\title{
Hallux Limitus y su relación con el pie pronado como factor etiológico
}

\author{
Hallux Limitus and relationship with the foot pronated \\ as etiological factor \\ Raúl BLAZQUez ViUdAS \\ Diplomado en Podología. Colaborador Universidad Complutense de Madrid.
}

\begin{abstract}
Correspondencia:
Raúl Blázquez Viudas.

C/ La Albera, $n^{\circ} 17$ a, $1^{\circ} \mathrm{G}$.

30120. El Palmar

Murcia

Fecha de recepción: 6 de octubre de 2009

Fecha de aceptación: 20 de diciembre de 2009

El autor declara no tener ningún tipo de interés económico o comercial.
\end{abstract}

\section{RESUMEN}

La primera articulación metatarsofalángica es muy importante en la fase propulsiva de la marcha, de ahí su importancia biomecánica. Por eso es muy importante estudiar las patologías que afectan a dicha articulación. Una de las patologías que más se dan en la primera articulación metatarsofalángica es el Hallux Limitus, de ahí la importancia de comprender mejor su clínica, estadios y etiología, sobre todo los factores etiológicos de origen biomecánico como es la hiperpronación, ya que es el que más prevalencia tiene.

Por eso, en el presente artículo se pretende hacer una revisión bibliografica y confirmar si el exceso de pronación es una causa de Hallux Limitus.

Palabras clave: Hallux Limitus, primera articulación metatarsofalángica, pie pronado, Mecanismo de Windlass.

\begin{abstract}
:
The first metatarsophalangeal joint is very important in the propulsive phase of gait, hence the importance of biomechanics. It is therefore very important to study the conditions affecting this joint. One of the pathologies that occur over the first metatarsophalangeal joint is hallux limitus, therefore the importance of better understanding their clinical stages, and etiology, especially the etiologic factors of biomechanical origin as overpronation, as is the most common is.

Therefore, this article seeks to make a literature review and confirm whether the excess pronation is a cause of hallux limitus.
\end{abstract}

Key Words: Hallux Limitus, first metatarsophalangeal joint, pronated foot, Windlass Mechanism.

Sumario: 1. Introducción y definición. 2. Clasificación y estadios del Hallux Limitus. 3. Etiología. 4. Relación del Hallix Limitus con el aumento de la pronación. 5. Conclusiones. 6. Bibliografía.

\section{Referencia normalizada:}

Blázquez Viudas, R. Hallux Limitus y su relación con el pie pronado como factor etiológico. Revista Internacional de Ciencias Podológicas 2011; 5 (1): 21-27. 


\section{INTRODUCCIÓN Y DEFINICIÓN}

El Hallux Limitus es una alteración degenerativa de la primera articulación metatarsofalángica, que se define clínicamente como una disminución de la flexión dorsal del hallux por debajo de $65^{\circ}$ en descarga ${ }^{(1-6)}$.

Las patologías y deformidades de la articulación metatarsofalángica del hallux constituyen las lesiones con mayor incidencia y prevalencia en las consultas de podología, la incidencia de lesiones como el Hallux Rigidus según la bibliografía revisada es de 1 de cada 45 individuos de más de 50 años. Siendo la segunda articulación, tras la rodilla, en severidad degenerativa del cartílago articular de toda la extremidad inferior ${ }^{(6)}$.

El rango de movimiento de la primera articulación metatarsofalángica es de $65^{\circ}$, aunque algunos autores hablan que puede llegar hasta los $90^{\circ}$, de los cuales entre $43^{\circ}-48^{\circ}$ respecto al primer metatarsiano y entre $20^{\circ}-30^{\circ}$ son respecto al suelo en carga, aunque algunos autores hablan entre valores de $50^{\circ}$ a $90^{\circ}$ de flexión dorsal $^{(1,4,5,7,8)}$.

La dorsiflexión de la primera articulación metatarsofalángica es necesaria para estabilizar el pie durante la fase propulsiva de la marcha ${ }^{(3,4,9,10,11,12)}$ y activar el Mecanismo de Windlass $(13,14,15,16,17,18)$ por eso de este modo, una disminución de la flexión dorsal de la primera articulación metatarsofalángica, se cree que es causante de una función anormal del pie y por tanto, de patología como el Hallux Rigidus, como se demuestra en varios artículos y estudios ${ }^{(19,20)}$.

\section{CLASIFICACION Y ESTADIOS DEL HALLUX LIMITUS}

Para comprender mejor esta deformidad el Hallux Limitus se clasifica en:

- Hallux Limitus Funcional: es cuando la articulación metatarsofalángica del hallux presenta un rango de movimento en flexión dorsal menor de $65^{\circ}$ en dinámica.

- Hallux Limitus Estructural: es cuando la articulación metatarsofalángica del hallux presenta un rango de movimento en flexión dorsal menor de $20^{\circ}$ a $30^{\circ}$ en estática.
El Hallux Limitus, tiene cuatro fase o estadios de progresión de la deformidad donde, va pasando de ser, según el grado de artrosis y anquilosis de la articulación metatarsofalángica del hallux, de Hallux Limitus funcional donde solo existe limitación en dinámica, Hallux Limitus Funcional donde además de en dinámica, también hay disminución del rango de flexión dorsal en estática, y por ultimo en Hallux Rigidus, que es el estadio mas avanzado y donde existen menos de $10^{\circ}$ de flexión dorsal de la primera articulación metatarsofalángica. Estos son los estadios clasificados por el rango de disminución de la flexión dorsal del hallux en grados, a continuación se muestra brevemente los estadios según el grado de dolor, anquilosis y deformidad ${ }^{(19,20)}$ :

- Estadio 1: Estadio de Hallux Limitus Funcional (Figura 1)

- Hallux equino o extensus.

- Subluxación plantar de la falange proximal del hallux.

- Metatarsus Primus Elevatus.

- La dorsiflexión puede ser normal en descarga, pero las fuerzas reactivas del suelo elevan el primer metatarsiano y lo elevan.

- No hay cambios degenerativos a nivel radiológico.

- Hiperextensión de la articulación interfalángica del hallux.

- Pie pronado.

- Estadio 2: Estadio de adaptación articular (Figura 2)

- Allanamiento de la primera cabeza metatarsal.

- Defectos/Lesiones osteocondrales.

- Erosión del cartílago.

- Dolor al final del rango de movimiento.

- Disminución pasiva del rango de movimiento.

- Pequeña exostosis dorsal.

- Abrasión/ Erosión subcondral. 


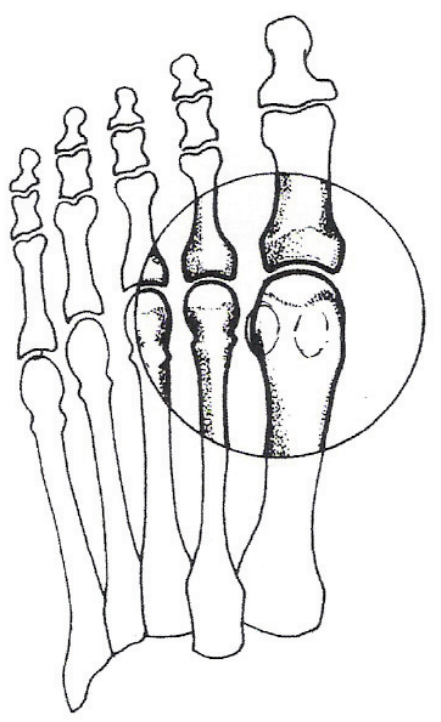

Figura 1: Estadio de Hallux Limitus Funcional

- Estadio 3: Estadio de estabilización de la artrosis (Figura 3)

- Severo allanamiento de la primera cabeza metatarsal.

- Osteofitosis, particularmente a nivel dorsal.

- Disminución y asimetría del espacio articular.

- Degeneración del cartílago articular.

- Erosión, excoriación de la articulación.

- Crepitación articular.

- Quistes subcondrales.

- Dolor en todo el rango de movimiento.

- Estadio 4: Estadio de anquilosis (Figura 4)

- Destrucción del espacio articular.

- Exuberante osteofitosis que pinza el espacio articular y la cápsula.

- Menos de $10^{\circ}$ de flexión dorsal.

- Deformidad y/o mal alineamiento.

- Anquilosis total.

- Inflamación local secundaria a inflamación de la piel o bursitis causada por la osteofitosis.

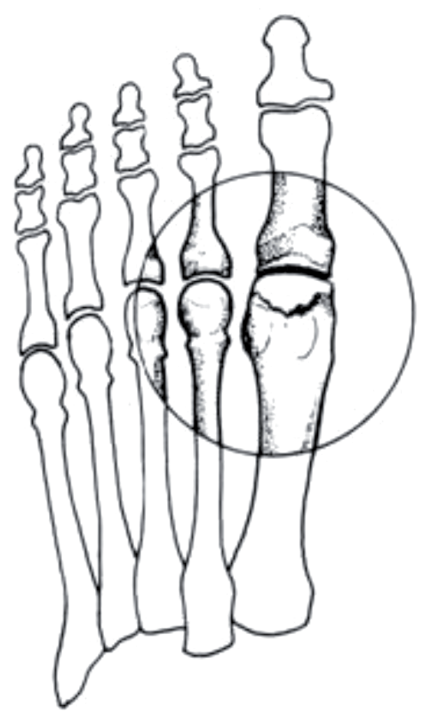

Figura 2: Estadio de adaptación articular

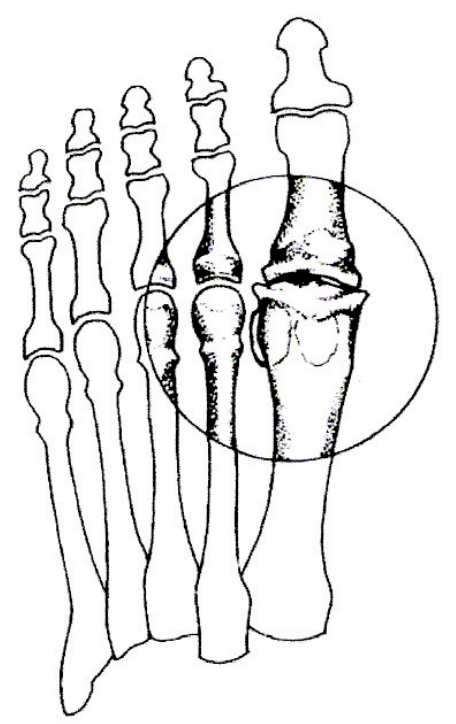

Figura 3: Estadio de estabilización de la artrosis 


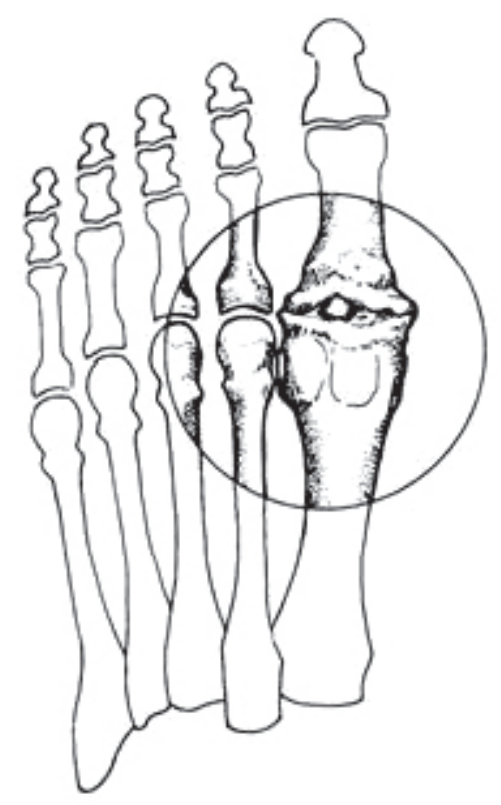

Figura 4: Estadio de anquilosis

\section{ETIOLOGIA}

Los principales factores etiológicos del Hallux Limitus se encuentran resumidos en la siguiente tabla ${ }^{(19)}$. (Tabla 1).

Dentro de todos los factores etiológicos del Hallux Limitus resumidos en la Tabla 1, en el presente artículo, nos vamos a centrar en la hiperpronación como factor etiológico de origen biomecánico, ya que, como se muestran en varios estudios sobre el tema, como el de Grady y cols. ${ }^{(8)}$, que es un estudio retrospectivo sobre 772 pacientes, en el cual se muestra que el $45 \%$ de los pacientes presentaban como factor etiológico alguna causa biomecánica, en el cual el 9,6\% eran por una excesiva pronación del pie y un $35,4 \%$ causado por un metatarsus primus elevatus como factor etiológico del hallux valgus.

El factor etiológico biomecánico también se confirma en los estudios de Roukis ${ }^{(21)}$ y en el de Kalish y cols. ${ }^{(22)}$, donde concluye que una disminución de la flexión dorsal de la primera articulación metatarsofalángica causado por un metatarsus primus elevatus produce una artrosis degenerativa de la primera articulación metatarsofalángica.

\section{RELACIÓN DEL HALLUX LIMITUS CON EL AUMENTO DE LA PRONACIÓN}

Revisando los estudios mencionados con anterioridad, se observa la importancia de indagar más en profundidad sobre los factores etiológicos de origen biomecánico, y sobre todo el exceso de pronación, que es el que aparece en la mayoría de los casos.

Por eso, estudiando el exceso de pronación se observa que los elementos estabilizadores más importantes del primer radio son el Peroneo Lateral Largo y la Fascia Planar, de ahí que, cuando se produce un exceso de pronación se acompaña de una hipermovilidad mas una dorsiflexión e inversión del primer radio, debido al aumento de las fuerzas reactivas del suelo sobre el primer metatarsiano y la falta de fulcro del peroneo lateral largo, que actua como elemento estabilizador del primer radio, produciendo todo esto una subluxación de la primera articulación metatarsofalángica. Esta hipermovilidad es el factor predisponerte mas importante que causa hallux limitus y hallux abductus valgus ${ }^{(2)}$.

También, cuanto mayor sea la magnitud del momento pronador subtalar, o mayor el momento de dorsiflexión del metatarsiano, mayor será la resistencia del hallux frente a la dorsiflexión. Cuanto mayor sea la resistencia a la dorsiflexión del hallux, mayores serán las tensiones dentro de las estructuras anatómicas a nivel plantar ${ }^{(2)}$.

Cuando se rompe el equilibrio de fuerzas que se ejercen sobre el primer radio las fuerzas reactivas del suelo y las estructuras que lo estabilizan, la columna interna se vuelve más inestable. Esto se produce en pies con soporte de peso, con un alto momento pronador de la articulación subastragalina, una cantidad elevada de fuerza bajo la cabeza metatarsal del primer metatarsiano y del hallux, o en un arco longitudinal interno disminuido, donde las fuerzas tensiles dentro de la aponeurosis plantar son muy grandes.

La inestabilidad de la columna interna favorece el desarrollo de alteraciones en la posición del primer metatarsiano. Ambos factores provocan una pérdida de la eficacia del mecanismo de Windlass ${ }^{3}$ y Jose).

Sobre la acción y repercusión del Mecanismo de Windlass, el estudio de Kappel-Bargas 
Tabla 1: Factores etológico del Hallux Limitus ${ }^{(9)}$

(*) Articulación metatarsofalángica

\begin{tabular}{|c|c|}
\hline Traumático & $\begin{array}{ll}\text { - } & \text { Lesión osteocondral } 1^{\circ} \text { AMTF }\left(^{*}\right) \\
\text { - } & \text { Fractura intraarticular } 1^{\circ} \text { AMTF } \\
\text { - } & \text { Disfunción de sesamoideos } \\
\text { Mala unión secundaria a una fractura } \\
\text { del } 1^{\circ} \text { metatarsiano }\end{array}$ \\
\hline Anatómico/Estructural & $\begin{array}{l}\text { - Longitud anormal de la falange proxi- } \\
\text { mal del hallux } \\
\text { - Longitud anormal del } 1^{\circ} \text { metatarsiano }\end{array}$ \\
\hline Metabólico & $\begin{array}{l}\text { - Condiciones artríticas que afecten a la } \\
\text { - } 1^{\circ} \text { AMTF } \\
\text { Defectos osteocondrales del } 1^{\circ} \mathrm{AMTF}\end{array}$ \\
\hline Biomecánico & $\begin{array}{l}\text { - Hipermovilidad del primer radio } \\
\text { - } \quad \text { Pronación excesiva }\end{array}$ \\
\hline Neuromuscular & $\begin{array}{l}\text { - Intrínseco o Extrínseco disbalance mus- } \\
\text { cular que afecte al } 1^{\circ} \text { radio }\end{array}$ \\
\hline Post-Quirúrgico $1^{\circ}$ radio & $\begin{array}{l}\text { - Exceso de elevación del } 1^{\circ} \text { metatarsiano } \\
\text { - } \\
\text { Excesivo alargamiento del } 1^{\circ} \text { metatar- } \\
\text { - } \text { Fibrosis excesiva } \\
\text { - } \text { Disfunción de sesamoideos } \\
\text { - Mal alineamiento de la } 1^{\circ} \mathrm{AMTF} \\
\text { - Inmovilización prolongada de la } 1^{\circ} \\
\text { AMTF }\end{array}$ \\
\hline
\end{tabular}

y cols. ${ }^{(16)}$ confirma que este mecanismo se activa durante la extensión pasiva de la primera articulación metatarsofalángica, y que durante la marcha, en la activación del mecanismo de Windlass se produce una inversión del calcáneo y que en pies con medialización del ASA y Navicular Drop positivo, como muestra Aquino y cols. ${ }^{(18)}$ en su estudio, intervienen en un mecanismo de Windlass ineficiente y en consecuencia con un hallux limitus.

En referencia a la función y consecuencias biomecánicas del Mecanismo de Windlass respecto al Hallux Limitus, existen multitud de estudios en dinámica que muestran la relación del Hallux Limitus sobre la posición del pie en la marcha, uno de ellos es el de Harra- dine y Bevan, ${ }^{(4)}$ en el cuál confirman que a mayor eversión del retropié menor flexión dorsal de la primera articulación metatarsofalángica, mostrando así que existe una relación entre el Hallux Limitus Funcional y la pronación, pero en este estudio, solo lo valora a nivel del retropié. Smith y cols. ${ }^{(9)}$ confirman, en otro estudio que cuñas de $5^{\circ}$ de valgo a nivel del retropié aumentan la flexión dorsal de la primera articulación metarsofalágica en dinámica, como en el estudio de Munuera y cols. ${ }^{(7)}$ donde utilizan ortesis para disminuir la pronación, demostrando que hay una relación entre el aumento pronación del pie y el hallux limitus.

Pero en cambio, en estática no hay muchos artículos, de ahí la importancia de estos tipos 
de estudio, como el estudio de Paton ${ }^{(15)}$ que demuestra que, existe una relación entre la disminución de la flexión dorsal del hallux en estatica, Hallux Limitus Estructural y la pronación a nivel de la articulación mediotarsiana con la prueba del Navicular Drop en estática. Y solo esta el estudio de Munteanu y Bassed ${ }^{(13)}$, que es el único en la bibliografía revisada que utiliza el Índice Postural del Pie, para observar la relación entre la postura del pie y el hallux limitus en estática y con ortesis invertidas, mostrando que a mayor pronación del pie, menor flexión dorsal de la primera articulación metatarsofalángica. En este ultimo estudio, es importante recalcar, que es el único de todos los revisados que utiliza para el mismo una de las pocas, o la única prueba validada científicamente que existe en la podología como es el Índice Postural del Pie ${ }^{(23-26)}$.

\section{CONCLUSIONES}

Una vez revisados todos los estudios consultados, se observa la asociación existente entre el hallux limitus y la hiperpronación, como principal factor etiológico.

La mayoría de los estudios revisados, se realizan en dinámica comparando el hallux limitus con pruebas como el navicular drop, el valgo de retropié o con ortesis plantares con cuñas en valgo o invertidas, mostrando una relación de la deformidad con un exceso de pronación en dinámica, pero también se evidencia la falta de estudios que muestren la relación con la estática o posición del pie, para sí poder tener una relación causa efecto mas alta, dejando así la puerta abierta a posibles nuevos estudios sobre el tema.

\section{BIBLIOGRAFIA}

1. Pascual Gutiérrez R, López Ros P, Alonso Montero C, Chicharro Luna E, Bustos García MJ. Patomecanica del primer radio. Revista Española de Podología 2001; XII (6): 323-333.

2. Kirby KA. Foor and lower extremity biomechanics II. Ed, precision Intricast,Inc. Arizona 2002: 139152.

3. Valmassy RL. Pathomechanics of the lower extremity function. In: Valmassy RL, ed. Clinical Biomechanics of the Lower Extremities. St Louis: Mosby, 1996: 59-84.

4. Harradine PD, Bevan LS. The effect of rearfoot eversion on maximal hallux dorsiflexion. J Am Podiatr Med Assoc 2000, 90(8): 390-393.

5. Van Gheluwe B, Dananberg HJ, Hagman F, Vanstaen K. Effects of hallux limitus on plantar foot pressure and foot kinematics during walking. J Am Podiatr Med Assoc 2006, 96(5): 428-436.

6. Monzon F, Soriano F, Montoro A, Calpena L, Meroño F, Pascual Gutiérrez R, Alonso Montero C, López Ros P, Bustos García MJ, Chicharro Luna R. Patomecanica del hallux limitus. Revista Española de Podología 2002; XIII (1): 13-18.

7. Munuera PV, Domingez G, Palomo IC, Lafuente G. Effects of rearfoot-controlling orthotic treatment on dorsiflexion of the hallux in feet with abnormal subtalar pronation. J Am Podiatr Med Assoc 2006, 96(4): 283-289.

8. Grady JF, Axe Tm, Zager EJ, Sheldon LA. A retrospective analysis of 772 patients with hallux limitus. J Am Podiatr Med Assoc 2002, 92(2): 102-108.

9. Smith C, Spooner SK, Fletton JA. The effect of 5-degree valgus and varus rearfoot wedging on peak hallux dorsiflesion during gait. J Am Podiatr Med Assoc 2004 94(6): 558-564.

10. Munteanu SE, Bassed AD. Effect of foot posture and inverted foot orthoses on hallux dorsiflexion. $\mathrm{J}$ Am Podiatr Med Assoc 2006, 96(1): 32-37.

11. Payne C, Chuter V, Miller K. Sensitivity and Specifity on the funtional hallux limitus test to predictor foot funtion. J Am Podiatr Med Assoc 2002, 92(5): 269-271.

12. Root M, Orien W, Weed J. Normal and Abnormal Function of the Foot, 358, Clinical Biomechanics Corp, Los Angeles, 1977.

13. Munteanu SE, Bassed AD. Effect of foot posture and inverted foot orthoses on hallux dorsiflexion. J Am Podiatr Med Assoc 2006, 96(1): 32-37.

14. Fuller EA. The Windlass mechanims of the foot. J Am Podiatr Med Assoc 2000, 90(1): 35-46.

15. Paton JS. The relationship between navicular drop and first metatarsophalangeal joint motion. J Am Podiatr Med Assoc 2006, 96(4): 313-317. 
16. Kappel-Bargas A, Woolf DR, Cornwall MW, McPoil TG. The windlass mechanism during normal walking and passive first metatarsalphalangeal joint extension. Clinical Biomechanics 1998, 13 (3): 190-194.

17. Carlson RE, Fleming LL, Hutton WC. The Biomechanical relationship between the tendoachilles, plantar fascia and metatarsophalangeal joint dorsiflexion angle. Foot \& Ankle International 2000, 21 (1): 18-24.

18. Aquino A, Payne C. Funtion of the windlass mechanism en excessively pronated feert. J Am Podiatr Med Assoc 2001, 91(5): 245-250.

19. Vanore JV, Christensen JC, Kravitz SR, Schuberth JM, Thomas JL. Weil LS. Diagnosis and treatment of first metatarsophalangeal joint disorders. Section 2: Hallux Rigidus. The Journal of The Foot \& Ankle Surgery 2003 42(3): 124-136.

20. Camastra CA. Halux Limitus and Hallux Rigidus. Clinical Examination, Radiographic Findings and Natural History. Clinics in Podiatrics Medicine and Surgery 1996 13(3): 442-448.

21. Roukis TS, Metatarsus primus elevatus in hallux rigidus. J Am Podiatr Med Assoc 200, 95(3): 221228.

22. Kalish SR, Willis FB. Hallux limitus and dynamic splinting: a retrospective serie. The Foot and Ankle Journal 2009, 2 (4): 1-4.

23. Pascual Gutiérrez R, López Ros P, García Campos J, Redmond A, Monzo Pérez FE, Herrera Fernández JM. Foot Posture Index. Salud del pie. Revista andaluza de podología 2008; 2: 4-14.

24. Scharfbilling R, Evans Am, Copper AW, Williams M, Scutter S, Lasiello H, Redmond A. Criterion validation of four criteria of the foot posuture index. J Am Podiatr Med Assoc 2004, 94(1): 31-38.

25. Redmond A, Crane YZ, Menz HB. Normative values for the foot posture index. Journal of Foot and Ankle Research 2008, 1 (6): 1-9.

26. Evans Am, Copper AW, Scharfbilling R, Scutter S, Williams M. Releability of the foot posture index and traditional measures of foot position. J Am Podiatr Med Assoc 2003, 93(3): 203-213. 